— la viabilité des portées réduites est légèrement inférieure à celle des portées non modifiées. La différence est plus grande pour les portées fortement réduites;

- les portées choisies pour adopter des lapereaux ont une viabilité nettement supérieure à la viabilité moyenne de l'échantillon non modifié $(87 \mathrm{p}$. roo contre 78 p. Ioo);

- la viabilité après adoption dépend à la fois du nombre de lapereaux ajoutés et de la taille de la portée d'accueil. Les meilleurs résultats sont obtenus en ajoutant un seul lapereau par portée.

\title{
PROBLÈMES POSÉS PAR IA PRODUCTION DE FOURRURE DE LAPIN : BIBLIOGRAPHIE ET PROJET D'ÉTUDE
}

\section{J. I. VRILLON, H. DE ROCHAMBEAU}

Domaine expérimental du Magneraud, Boîte Postale 52, I77oo Surgeres Chaire de Zootechnie, INA Paris-grignon, I6, nue C. Bernard Paris $V^{\mathrm{e}}$

L'évolution récente des systèmes de production du lapin n'a pas permis de valoriser la peau en vue de l'utilisation de la fourrure, or cette peau peut constituer un supplément de revenu important pour le producteur (de 0,3 à $6 \mathrm{~F}$ la peau). En France la plus grosse partie de la production (6o p. Ioo) est réalisée chez 98,5 p. Ioo des éleveurs de très petits troupeaux (moins de Io mères) appliquant mal les récentes découvertes en matière d'alimentation et de technique d'élevage hors sol. Cette population d'éleveurs est notre cible dans la recherche d'une meilleure valorisation de la peau. Nous avons cherché quel est le meilleur critère de la qualité d'une fourrure. La surface est retenue dans les pays de l'Est européent. La couleur, déterminée par 6 loci comporte un grand nombre de variations possibles et le travail de fixation reste à faire, la mesure de la densité pose un problème technique. La finesse et la longueur du poil semblent jouer en faveur du choix d'un animal présentant la mutation Rex. Nous nous proposons d'approfondir les recherches en vue d'associer à terme, en un même animal, à des performances zootechniques convenables une bonne qualité de toison compatible avec une technologie artisanale de traitement. 